\title{
FUNCTIONAL EQUATIONS IN TOTAL NEGATION
}

\author{
T. B. M. MCMASTER and C. R. TURNER
}

(Received 26 October 1998; revised 9 September 1999)

Communicated by J. A. Hillman

\begin{abstract}
It is known that the only topological invariants $P$ for which $\operatorname{anti}(P)=\operatorname{anti}{ }^{2}(P)$, anti( ) denoting Bankston's total negation operator, are those which are determined purely by the cardinality of the underlying point-set. We examine equations of the form anti ${ }^{n}(P)=$ anti $^{n}$ (not $P$ ), reaching similar conclusions for $n \leq 2$ but weaker ones for $n \geq 3$. A corresponding investigation for total negation within a constraint is initiated.
\end{abstract}

2000 Mathematics subject classification: 54A25, 54B05, 54D99.

\section{Introduction}

The total negation operator anti( ) was introduced by Bankston in [1]. For each topological invariant $P$ it generates another, anti $(P)$, by declaring a space $X$ to be anti $(P)$ provided that, whenever $Y$ is both a $P$ space and a subspace of $X$, then the cardinality of the point-set $Y$ is such that every topology that can be imposed upon it renders it a $P$ space. The best known instance, and one of the historical roots from which the general theory grew, is that an anti(compact) space is one that has no compact subspaces excepting, inevitably, the finite ones. There is a substantial and growing literature both on the general process and on important special cases: see, for example, $[2-5,10,11,13]$.

Let us call an invariant $P$ cardinally decisive if there do not exist a $P$ space and a not $P$ space whose underlying point-sets have the same cardinality. Matier and McMaster noted [4] that these are the only properties $P$ for which anti $(P)$ and anti(anti $(P))$ coincide; further, for cardinally decisive $P$, all spaces are anti $(P)$. Our purpose in

(C) 2000 Australian Mathematical Society $0263-6115 / 2000 \$ A 2.00+0.00$ 
this article is to explore other such coincidences, especially the equations anti $(P)=$ anti(not $P)$, anti(anti $(P))=\operatorname{anti}(\operatorname{anti}($ not $P))$ and so on. We shall use the natural notation $\operatorname{anti}^{n}()$ for the iterates of Bankston's operator, defined by $\operatorname{anti}^{0}(P)=P$, $\operatorname{anti}^{n+1}(P)=\operatorname{anti}\left(\operatorname{anti}^{n}(P)\right)$ if $n \geq 0$. Further notations which will facilitate the discussion are that for a given invariant $P$,

$\operatorname{spec}(P)=\{\lambda: \lambda$ is a non zero cardinal number, and every space whose point-set has cardinality $\lambda$ is a $P$ space $\}$,

$\operatorname{ind}(P)=\{\lambda: \lambda$ is a non zero cardinal number, and some but not all spaces whose point-sets have cardinality $\lambda$ are $P$ spaces\},

$\mathscr{U}=$ the 'universal class' of all topological spaces (modulo homeomorphism).

\section{Results}

The following results, taken from [4], are easily verified.

LEMMA 1. Let $P$ be a topological invariant. Then

(i) the non anti $(P)$ spaces are precisely those that have a $P$ subspace $Y$ whose cardinality $|Y| \in$ ind $(P)$;

(ii) if a space $X$ is both $P$ and anti $(P)$ then $|X| \in \operatorname{spec}(P)$;

(iii) we have anti $(P)=\mathscr{U}$ if and only if ind $(P)=\emptyset$;

(iv) $\operatorname{spec}(\operatorname{anti}(P)) \cap \operatorname{ind}(P)=\emptyset$.

THEOREM 2. Let $P$ be a topological invariant. Then the following are equivalent:

(i) $\operatorname{anti}(P)=\operatorname{anti}(\operatorname{not} P)$;

(ii) $\operatorname{anti}(P)=\operatorname{anti}($ not $P)=\mathscr{U}$;

(iii) $\operatorname{ind}(P)=\emptyset$.

PrOOF. Since ind $(P)=$ ind(not $P$ ), the implication (iii) implies (ii) derives from Lemma 1 while (ii) implies (i) is immediate. Now suppose if possible that there is a property $P$ for which anti $(P)$ and anti(not $P$ ) coincide but ind $(P)$ is not empty. Let $\lambda$ be the least member $\min (\operatorname{ind}(P))$ of ind $(P)$ and choose any space $X$ of cardinality $\lambda$ that is 'minimal' in the sense that every $\lambda$-element subspace of $X$ is homeomorphic to $X$. (Matthews $[6,7]$ calls such spaces strongly quasi-minimal. A discrete or a trivial space on $\lambda$-many points would suffice here.) Let us consider whether $X$ is anti $(P)$ or not.

If $X$ is anti $(P)=$ anti(not $P$ ) then it cannot be $P$ : else the fact that $X$ is a subspace of itself implies that its cardinality $\lambda$ belongs to $\operatorname{spec}(P)$, which is not how $\lambda$ was chosen. Yet the same pattern of argument shows that $X$ cannot be not $P$. The contradiction forces us to conclude that $X$ is not anti $(P)$. 
Choose therefore a $P$ subspace $Y$ of $X$ whose cardinality $|Y|$ belongs to ind $(P)$. Since $|Y| \leq \lambda=\min (\operatorname{ind}(P))$ we find $|Y|=\lambda$ and deduce that $Y$ and $X$ are homeomorphic. Hence $X$ is $P$. However, a similar argument will establish that $X$ is not $P$ also, giving a final contradiction.

Referring back to the Matier and McMaster result quoted above, it is not true that $\operatorname{anti}^{2}(P)=\operatorname{anti}^{2}(\operatorname{anti}(P))$ if and only if $P$ is cardinally decisive: for instance [5], anti $^{2}\left(\right.$ first countable) and anti ${ }^{3}$ (first countable) both coincide with $\mathscr{U}$. In contrast, Theorem 2 remains valid for the second total negation.

THEOREM 3. Let $P$ be a topological invariant. Then the following are equivalent:

(i) $\operatorname{anti}^{2}(P)=\operatorname{anti}^{2}(\operatorname{not} P)$;

(ii) $\operatorname{anti}^{2}(P)=\operatorname{anti}^{2}($ not $P)=\mathscr{U}$;

(iii) ind $(P)=\emptyset$.

ProOF. We shall merely show that (i) implies (iii), since the rest of the demonstration is trivial. Suppose if possible that $\operatorname{anti}^{2}(P)=\operatorname{anti}^{2}($ not $P$ ) but that ind $(P) \neq \emptyset$. Put $\lambda=\min (\operatorname{ind}(P))$ and choose a strongly quasi-minimal space $X$ on $\lambda$-many points. We may suppose that $X$ is $P$ else, just interchange $P$ and not $P$, and we consider whether $X$ is $\operatorname{anti}^{2}(P)$ or not.

Suppose that $X$ is anti ${ }^{2}(P)$. If it is not anti(not $P$ ) then choose $B \subseteq X$ such that $B$ is not $P$ but $|B| \in \operatorname{ind}($ not $P)=\operatorname{ind}(P)$. The minimality of $\lambda$ shows that $|B|=\lambda$ and the strong quasi-minimality of $X$ gives $B$ and $X$ homeomorphic. Thus $X$ is not $P$, contrary to assumption, and we deduce that $X$ is anti(not $P$ ). Yet $X$ is also anti(anti(not $P)$ ) and a subspace of itself, so $\lambda=|X| \in \operatorname{spec}(\operatorname{anti}($ not $P))$. This is easily seen to be incompatible with $\lambda \in \operatorname{ind}(\operatorname{not} P)$.

Consequently, $X$ must not be $\operatorname{anti}^{2}(P)$, and we can choose $Y \subseteq X$ such that $Y$ is anti $(P)$ but $|Y| \in \operatorname{ind}(\operatorname{anti}(P))$. Now if $|Y|=\lambda$ we get $X$ and $Y$ homeomorphic, whence $X$ would be both $P$ and anti $(P)$, giving the contradiction $\lambda=|X| \in \operatorname{spec}(P)$. Therefore $\mu=|Y|<\lambda$. Since $\mu \in \operatorname{ind}(\operatorname{anti}(P))$ there is a space $Z$ on $\mu$-many points such that $Z$ is not anti $(P)$, and there is $W \subseteq Z$ with $W$ being $P$ but $|W| \in \operatorname{ind}(P)$. Yet $|W|<\lambda$, so the choice of $\lambda$ yields a conclusive contradiction.

No wholesale extension of Theorem 3 to the third anti-property is possible since, for instance, it is known [5] that anti ${ }^{3}$ (completely-separable) $=\operatorname{anti}^{3}$ (not completelyseparable) whereas ind(completely-separable) comprises all infinite cardinals. Notice, however, that $\operatorname{anti}^{3}$ (completely-separable) $=\mathscr{U}$, so this example does not invalidate the hypothesis that (i) and (ii) might remain equivalent for $\operatorname{anti}^{3}()$ in place of $\operatorname{anti}^{2}()$. This hypothesis is indeed correct for all higher iterates, as we shall now show. The bulk of the demonstration takes place in Lemma 5 below; here, an invariant $P$ is 
called monotone (following [4]) when $\operatorname{spec}(P)$ is a decreasing class of cardinals (in the sense that $\mu<\lambda, \lambda \in \operatorname{spec}(P)$ imply $\mu \in \operatorname{spec}(P))$ and also $\operatorname{spec}(P) \cup$ ind $(P)$ is a decreasing class. We use the following observation [4] concerning monotonicity.

LEMMA 4. Hereditary invariants are monotone; in particular, anti $(P)$ is monotone for every $P$.

LEMMA 5. Let $P$ be a monotone invariant and $m \geq 2$ a positive integer. Then the following are equivalent:

(i) $\operatorname{anti}^{m}(P)=\operatorname{anti}^{m}($ not $P$ );

(ii) $\operatorname{anti}^{m}(P)=\operatorname{anti}^{m}($ not $P)=\mathscr{U}$.

PROOF. Suppose that $\operatorname{anti}^{m}(P)=\operatorname{anti}^{m}($ not $P) \neq \mathscr{U}$. From Lemma $1, \operatorname{ind}(P) \neq \emptyset$ so we may again select a space $X$ that is strongly quasi-minimal and of cardinality $\lambda=\min (\operatorname{ind}(P))$ and, without loss of generality, $X$ is a $P$ space. For each nonnegative integer $n$ let $p(n)$ denote the following composite assertion:

(i) all spaces on fewer than $\lambda$ points $\operatorname{are~anti~}^{n}(P)$;

(ii) if $n$ is even, $X$ is $\operatorname{anti}^{n}(P)$;

(iii) if $n$ is odd, $X$ is not $\operatorname{anti}^{n}(P)$;

(iv) $\lambda \in \operatorname{ind}\left(\operatorname{anti}^{n}(P)\right)$.

Clearly $p(0)$ is true. Consider now a non-negative integer $k$ for which both $0 \leq$ $k<k+1 \leq m-1$ and $p(k)$ are satisfied. We seek to establish $p(k+1)$.

If $|Y|=\mu<\lambda$ and $Z \subseteq Y$ and $Z$ is anti $^{k}(P)$, then $|Z| \leq \mu<\lambda$. We see from $p(k)$ that $|Z| \in \operatorname{spec}\left(\operatorname{anti}^{k}(P)\right)$ and so $Y$ is anti $\left(\operatorname{anti}^{k}(P)\right)=\operatorname{anti}^{k+1}(P)$. That gives us $p(k+1)(\mathrm{i})$.

If $k+1$ is even, then $k$ is odd and $p(k)$ tells us that $X$ is not antik ${ }^{k}(P)$. Any anti $^{k}(P)$ subspace of $X$ must have fewer points than $X$, because of the latter's strong quasi-minimality, so by $p(k)$ (i) its cardinality belongs to $\operatorname{spec}\left(\operatorname{anti}^{k}(P)\right)$. That is, $X$ is anti ${ }^{k+1}(P)$. Since Lemma 4 tells us that $\lambda=|X|$ must lie outside $\operatorname{spec}\left(\operatorname{anti}^{k+1}(P)\right)$, belonging as it does to ind $\left(\operatorname{anti}^{k}(P)\right)$, we deduce that $\lambda \in \operatorname{ind}\left(\operatorname{anti}^{k+1}(P)\right)$.

On the other hand, if $k+1$ is odd then $k$ is even and $X$ is anti ${ }^{k}(P)$. It is then easy to see that $X$ cannot be anti ${ }^{k+1}(P)$, and $\operatorname{so} \lambda \notin \operatorname{spec}\left(\operatorname{anti}^{k+1}(P)\right)$. If $\lambda$ were also to fail to belong to ind $\left(\operatorname{anti}^{k+1}(P)\right)$ then monotonicity would imply that ind $\left(\operatorname{anti}^{k+1}(P)\right)=\emptyset$, whence from Lemma 1 we derive $\mathscr{U}=\operatorname{anti}^{k+2}(P)=\operatorname{anti}^{k+3}(P)=\cdots=\operatorname{anti}^{m}(P)$, a contradiction. So $\lambda$ must lie in ind( $\left.\operatorname{anti}^{k+1}(P)\right)$. At this stage, all of $p(k+1)$ is confirmed.

The recursion establishes $p(m-1)$, from which we obtain parts (ii) and (iii) of $p(m)$ just as before; that is: 
However, the entire process may now be repeated on the invariant not $P$, working on the composite assertion $p^{\prime}(n)$ :

(i) all spaces on fewer than $\lambda$ points are anti ${ }^{n}($ not $P)$;

(ii) if $n$ is even, $X$ is not anti ${ }^{n}($ not $P$ );

(iii) if $n$ is odd, $X$ is antin $($ not $P$ );

(iv) $\lambda \in \operatorname{ind}\left(\operatorname{anti}^{n}(\operatorname{not} P)\right)$

to show that

(†) if $m$ is even, $X$ is not anti ${ }^{m}\left(\right.$ not $P$ ) and if $m$ is odd, $X$ is anti ${ }^{m}($ not $P$ )

Bearing in mind that anti $\mathrm{i}^{m}(P)=\operatorname{anti}^{m}($ not $P),(*)$ and $(\dagger)$ are irreconcilable and the demonstration is complete.

THEOREM 6. Let $P$ be a topological invariant, and $m$ a positive integer. Then the following are equivalent:

(i) $\operatorname{anti}^{m}(P)=\operatorname{anti}^{m}(\operatorname{not} P)$;

(ii) $\operatorname{anti}^{m}(P)=\operatorname{anti}^{m}($ not $P)=\mathscr{U}$.

PROOF. For $m=1$ and $m=2$ this was established in Theorems 2 and 3. For $m \geq 3$ we apply Lemma 5 to the (hereditary, therefore monotone) property anti $(P)$ and the positive integer $m-1$.

REMARK. Recent investigations (see $[8,9,12]$ ) have shown that many of the concepts and results of total negation remain viable if the entire discussion is constrained to take place within a specified class of topological spaces rather than the whole of $\mathscr{U}$. The potential benefits of such an exploration are evident since many topological programmes are, through choice or necessity, so constrained: for example, to $T_{2}$ spaces, or separable metrizable spaces, or infinite spaces. For our investigation of functional equations, the principal danger of so constraining appears to be the risk of losing strongly quasi-minimal spaces: for not only were these a key ingredient in the above arguments, but we can readily exhibit an example of constrained total negation, deficient in minimal spaces, in which the results of the present article fail.

EXAMPLE. It is known [6,7] that there is a family $\left(R_{n}, n \in N\right)$ of subspaces of the real line $R$ which form a 'strictly decreasing chain under embeddability' in the sense that $R_{m}$ is homeomorphic to a subspace of $R_{n}$ if and only if $m \geq n$, and for which each $R_{n}$ has cardinality c. Let the family $\mathscr{C}$ of topological spaces consist of (i) all subspaces of $R$ that have fewer than c-many points, and (ii) all superspaces of $R$ that have more than $\mathrm{c}$-many points, and (iii) $R, R_{1}, R_{2}, R_{3}, \ldots$ Also let $P=\left\{R_{1}, R_{3}, R_{5}, R_{7}, \ldots\right\}$. Arguing exclusively within the constraint $\mathscr{C}$, it 
is clear that $\operatorname{spec}(P)=\emptyset$ and ind $(P)=\{\mathbf{c}\}$, so no space on fewer than $\mathrm{c}$ points contains a $P$ space whose cardinality falls into ind $(P)$ but every space on $\mathrm{c}$ points or more does so. Thus, anti $P)=\{X \in \mathscr{C}:|X|<c\}$. The same argument shows that anti(not $P)=\{X \in \mathscr{C}:|X|<c\}$ whereas-compare Theorem 2-ind $(P)$ is not empty. It may be relevant that $\mathscr{C}$ is not a hereditary class since, frequently, we find that constrained total negation within a hereditary constraint resembles the behaviour of the 'unconstrained', classical theory more closely. It is at present unclear to what extent the assumption of hereditariness might guarantee the presence of minimal spaces in examples such as this one.

\section{References}

[1] P. Bankston, 'The total negation of a topological property', Illinois J. Math. 23 (1979), 241-252.

[2] N. Levine, 'On the equivalence of compactness and finiteness in topology', Amer. Math. Monthly 75 (1968), 178-180.

[3] J. Matier and T. B. M. McMaster, 'Total negation in general topology', Irish Math. Soc. Bull. 25 (1990), 26-37.

[4] __ 'Iteration of Bankston's 'anti'-operation', J. Inst. Math. and Comp. Sci. Math. Ser. 3 (1990), 31-35.

[5] —_ 'Total negation of separability and related properties', Proc. Roy. Irish Acad. Sec. A 90 (1990), 131-137.

[6] P. T. Matthews and T. B. M. McMaster, 'Families of spaces having prescribed embeddability order-type', Rend. Istit. Mat. Univ. Trieste 25 (1993), 345-352.

[7] _ 'Minimal spaces, maximal pre-antis', Rend. Istit. Mat. Univ. Trieste 25 (1993), 353-361.

[8] T. B. M. McMaster and C. R. Turner, 'Constrained total negation and its iteration', preprint (Queen's University of Belfast, 1998).

[9] — 'Total negation under constraint: pre-anti properties', preprint (Queen's University of Belfast, 1998).

[10] I. L. Reilly and M. K. Vamanamurthy, 'Compactness and finiteness', Colloq. Math. Soc. János Bolyai 23 (1978), 1033-1041.

[11] _ 'Some topological anti-properties', Illinois J. Math. 24 (1980), 382-389.

[12] C. R. Turner, Total negation of topological properties in constrained environments (Ph.D. Thesis, Queen's University of Belfast, 1997).

[13] A. Wilansky, 'Between $\mathrm{T}_{1}$ and $\mathrm{T}_{2}$ ', Amer. Math. Monthly 74 (1967), 261-266.

Pure Mathematics Department

Queen's University

Belfast BT7 1NN

Northern Ireland

e-mail: t.b.m.mcmaster@qub.ac.uk
School of Electrical and

Mechanical Engineering University of Ulster at Jordanstown

Northern Ireland e-mail: c.turner@ulst.ac.uk 